\title{
Nationwide Surveillance on Antimicrobial Resistance Profiles of Enterococcus faecium and Enterococcus faecalis Isolated from Healthy Food Animals in South Korea, 2010 to 2019
}

\author{
Mi Hyun Kim, Dong Chan Moon (), Su-Jeong Kim, Abraham Fikru Mechesso, Hyun-Ju Song, Hee Young Kang, \\ Ji-Hyun Choi, Soon-Seek Yoon $\mathbb{D}$ and Suk-Kyung Lim *
}

\author{
Bacterial Disease Division, Animal and Plant Quarantine Agency, 177 Hyeksin 8-ro, Gimcheon-si 39660, \\ Gyeongsangbuk-do, Korea; kimmh940301@naver.com (M.H.K.); ansehdcks@korea.kr (D.C.M.); \\ kimsujeong27@gmail.com (S.J.K.); abrahamf@korea.kr (A.F.M.); shj0211@korea.kr (H.J.S.); \\ kanghy7734@korea.kr (H.Y.K.); wlgus01@korea.kr (J.-H.C.); yoonss24@korea.kr (S.-S.Y.) \\ * Correspondence: imsk0049@korea.kr; Tel.: +82-54-912-0738
}

check for updates

Citation: Kim, M.H.; Moon, D.C.; Kim, S.-J.; Mechesso, A.F.; Song, H.-J.; Kang, H.Y.; Choi, J.-H.; Yoon, S.-S.; Lim, S.-K. Nationwide Surveillance on Antimicrobial Resistance Profiles of Enterococcus faecium and Enterococcus faecalis Isolated from Healthy Food Animals in South Korea, 2010 to 2019. Microorganisms 2021, 9, 925. https://doi.org/ 10.3390/microorganisms 9050925

Academic Editor: Jens André Hammerl

Received: 7 April 2021

Accepted: 23 April 2021

Published: 26 April 2021

Publisher's Note: MDPI stays neutral with regard to jurisdictional claims in published maps and institutional affiliations.

Copyright: (c) 2021 by the authors. Licensee MDPI, Basel, Switzerland. This article is an open access article distributed under the terms and conditions of the Creative Commons Attribution (CC BY) license (https:// creativecommons.org/licenses/by/ $4.0 /)$.

\begin{abstract}
Intestinal commensal bacteria are considered good indicators for monitoring antimicrobial resistance. We investigated the antimicrobial resistance profiles and resistance trends of Enterococcus faecium and Enterococcus faecalis isolated from food animals in Korea between 2010 and 2019. E. faecium and E. faecalis, isolated from chickens and pigs, respectively, presented a relatively high resistance rate to most of the tested antimicrobials. We observed high ciprofloxacin (67.9\%), tetracycline (61.7\%), erythromycin $(59.5 \%)$, and tylosin (53.0\%) resistance in E. faecium isolated from chickens. Similarly, more than half of the E. faecalis isolates from pigs and chickens were resistant to erythromycin, tetracycline and tylosin. Notably, we observed ampicillin, daptomycin, tigecycline and linezolid resistance in a relatively small proportion of enterococcal isolates. Additionally, the enterococcal strains exhibited an increasing but fluctuating resistance trend $(p<0.05)$ to some of the tested antimicrobials including daptomycin and/or linezolid. E. faecalis showed higher Multidrug resistance (MDR) rates than E. faecium in cattle (19.7\% vs. $8.6 \%$, respectively) and pigs $(63.6 \%$ vs. $15.6 \%$, respectively), whereas a comparable MDR rate $(\approx 60.0 \%)$ was noted in E. faecium and E. faecalis isolated from chickens. Collectively, the presence of antimicrobial-resistant Enterococcus in food animals poses a potential risk to public health.
\end{abstract}

Keywords: antimicrobial resistance; E. faecium; E. faecalis; food animals; public health

\section{Introduction}

Enterococci are commensal bacteria of the gastrointestinal tract of animals and humans. They can also be detected in the environment and in foods of animal origin. They are considered emerging pathogens of humans and are often associated with invasive nosocomial infections [1]. Enterococci have emerged as good indicators of antibiotic resistance. They can acquire resistance genes from other bacteria, which can also spread to other commensal and pathogenic bacteria through horizontal transfer of mobile genetic elements [2]. Besides, the frequent use of antimicrobials in humans and animals select for resistant enterococci [3].

There are 55 enterococci species reported so far based on $16 S$ rDNA sequences. Enterococcus faecium and Enterococcus faecalis are the most commonly isolated species, accounting for more than $80 \%$ of isolates [4]. E. faecium and E. faecalis have become increasingly important pathogens worldwide because they are associated with life-threatening hospitalacquired infections, whereas the remaining Enterococcus spp. are infrequent causes of human clinical infections [3]. E. faecalis is the most pathogenic species, while E. faecium is commonly involved in the acquisition and transfer of antimicrobial resistance [5].

Food animals have been suggested as a possible intermediate vector in the transmission mode of antimicrobial-resistant enterococci. Cross-contamination of edible carcass 
tissues during the slaughter process represents a significant food safety hazard [6]. Tyson et al. [7] and Boehm et al. [8] demonstrated that more than $90 \%$ of food samples from animals are contaminated with Enterococcus at the slaughterhouse, predominantly with $E$. faecium and E. faecalis. Therefore, it is important to continuously evaluate the antimicrobial resistance profiles of E. faecium and E. faecalis strains found in food animals.

Monitoring the resistance profiles and temporal trends of antimicrobial resistance in Enterococcus species isolated from food animals provides useful information for understanding the level of resistance of gut microbial flora as well as for the empirical selection of antimicrobial agents to treat infected patients. Several researchers have reported the antimicrobial resistance profiles of Enterococcus isolated from food animals worldwide [9-14]. In South Korea (Korea), only a few studies have been conducted to determine the resistance profiles of E. faecium and E. faecalis isolates from food animals [15-20]. These studies were conducted in isolates collected from some parts of the country for a very short duration, and hence the resistance trends of the Enterococcus isolates remained unexplored. Thus, we performed this study to determine the antimicrobial resistance profiles and resistance trends of E. faecium and E. faecalis isolated from healthy cattle, pigs and chickens throughout Korea between 2010 and 2019.

\section{Materials and Methods}

\subsection{Bacterial Isolates}

A total of 3360 E. faecium isolates (572 isolates from cattle, 1385 from pigs, and 1403 from chickens) and 4218 E. faecalis isolates (910 isolates from cattle, 1556 from pigs, and 1752 from chickens) were obtained from 16 laboratories/centers participating in the Korean Veterinary Antimicrobial Resistance Monitoring System between 2010 and 2019. The isolates were recovered from the feces of animals in various slaughterhouses (Supplementary Table S1). No more than five fecal samples were collected from each farm. Sample processing and enterococcal isolation were performed according to Lim et al. [20] using buffered peptone water and Enterococcus agar media (Becton, Dickinson, Sparks, MD). Enterococcus species were identified by Polymerase Chain Reaction (PCR) [21] or matrix-assisted laser desorption ionization-time-of-flight mass spectrometry (MALDI-TOF, Biomerieux, Marcy L'Etoile, France). One isolate per sample was selected for the antimicrobial susceptibility test.

\subsection{Antimicrobial Susceptibility Study}

The antimicrobial susceptibility profiles of the enterococcal isolates were determined by the broth microdilution method [22], using commercially available antibiotic-containing plates (Sensititre, Trek Diagnostics, Cleveland, OH, USA). The following antimicrobials were tested: ampicillin $(1-16 \mu \mathrm{g} / \mathrm{mL})$, chloramphenicol $(2-32 \mu \mathrm{g} / \mathrm{mL})$, ciprofloxacin $(0.25-16 \mu \mathrm{g} / \mathrm{mL})$, daptomycin $(0.5-32 \mu \mathrm{g} / \mathrm{mL})$, erythromycin $(1-64 \mu \mathrm{g} / \mathrm{mL})$, florfenicol $(2-32 \mu \mathrm{g} / \mathrm{mL})$, gentamicin $(128-2048 \mu \mathrm{g} / \mathrm{mL})$, kanamycin $(128-2048 \mu \mathrm{g} / \mathrm{mL})$, linezolid $(0.5-16 \mu \mathrm{g} / \mathrm{mL})$, quinupristin/dalfopristin $(1-32 \mu \mathrm{g} / \mathrm{mL})$, salinomycin $(2-32 \mu \mathrm{g} / \mathrm{mL})$, streptomycin $(128-2048 \mu \mathrm{g} / \mathrm{mL})$, tetracycline $(2-128 \mu \mathrm{g} / \mathrm{mL})$, tigecycline $(0.12-2 \mu \mathrm{g} / \mathrm{mL})$, tylosin $(1-64 \mu \mathrm{g} / \mathrm{mL})$ and vancomycin $(2-32 \mu \mathrm{g} / \mathrm{mL})$. Enterococcus faecalis ATCC 29212 was used as a quality reference strain. The MIC values were interpreted according to the guidelines of the Clinical and Laboratory Standards Institute [22], the National Antimicrobial Resistance Monitoring System [23], and the Danish Integrated Antimicrobial Resistance Monitoring and Research Programme [24]. MIC50 and MIC90 values were defined as the lowest concentration of antimicrobials at which $50 \%$ and $90 \%$ of the isolates were inhibited, respectively. Isolates that were resistant to at least three subclasses of antimicrobials, excluding resistance to quinupristin/dalfopristin in E. faecalis, were considered multidrug-resistant. 


\subsection{Data Analysis}

Analysis of the antimicrobial resistance rates and Pearson correlation was conducted using Excel (Microsoft-Excel, 2016, Microsoft Corporation, Redmond, WA, USA) and Rex software (Version 3.0.3, RexSoft Inc., Seoul, Korea). $p$ values less than 0.05 were considered significant.

\section{Results}

\subsection{Antimicrobial Resistance}

In general, a more frequent occurrence of resistance to most of the tested antimicrobials was observed among the E. faecalis isolates compared with E. faecium (Table 1). More than $50 \%$ of the $E$. faecalis isolates were resistant to macrolides and tetracycline, whereas we noted a moderate resistance rate to these antimicrobials in E. faecium. Both enterococcal species demonstrated a very low resistance rate $(\leq 10.0 \%)$ to ampicillin, daptomycin, tigecycline, linezolid and salinomycin. However, all isolates were susceptible to vancomycin.

Table 1. Antimicrobial resistance rate in E. faecium $(n=3360)$ and E. faecalis $(n=4218)$ recovered from cattle, pigs, and chickens between 2010 and 2019 in Korea.

\begin{tabular}{|c|c|c|c|c|c|c|c|c|}
\hline \multirow[b]{3}{*}{ Antimicrobials } & \multicolumn{8}{|c|}{ Resistance Rate \% (Number of Isolates) } \\
\hline & \multicolumn{4}{|c|}{ E. faecium } & \multicolumn{4}{|c|}{ E. faecalis } \\
\hline & $\begin{array}{c}\text { Cattle } \\
(n=572)\end{array}$ & $\begin{array}{c}\text { Pigs } \\
(n=1385)\end{array}$ & $\begin{array}{l}\text { Chickens } \\
(n=1403)\end{array}$ & $\begin{array}{c}\text { Subtotal } \\
(n=3360)\end{array}$ & $\begin{array}{c}\text { Cattle } \\
(n=910)\end{array}$ & $\begin{array}{c}\text { Pigs } \\
(n=1556)\end{array}$ & $\begin{array}{l}\text { Chickens } \\
(n=1752)\end{array}$ & $\begin{array}{c}\text { Subtotal } \\
(n=4218)\end{array}$ \\
\hline Ampicillin & $0.3(2)$ & $0.1(1)^{\dagger}$ & $11.4(160)^{\#}$ & $4.8(163)$ & $0(0)$ & $0.1(1)$ & $0.1(2)$ & $0.1(3)$ \\
\hline Chloramphenicol & $2.3(13)^{*}$ & $6.9(95)^{+}$ & $18.8(264)^{\#}$ & $11.1(372)$ & $12.1(110)^{*}$ & $50.0(778)^{+}$ & $20.9(367)^{\#}$ & $29.8(1255)$ \\
\hline Ciprofloxacin & $23.1(132) *$ & $13.6(189)^{\dagger}$ & $67.9(953)^{\#}$ & $37.9(1274)$ & $7.9(72)^{*}$ & $13.7(213)^{\dagger}$ & $48.6(852){ }^{\#}$ & $27.0(1137)$ \\
\hline Daptomycin & $5.6(32)$ & $6.9(95)^{+}$ & $11.1(156)^{\#}$ & $8.4(283)$ & $1.3(12)$ & $0.6(10)$ & $0.6(10)^{\#}$ & $0.8(32)$ \\
\hline Erythromycin & $19.9(114)^{*}$ & $31.5(436)^{\dagger}$ & $59.5(835)^{\#}$ & $41.2(1385)$ & $20.4(186)^{*}$ & $67.1(1044)^{+}$ & $63.0(1104)^{\#}$ & $55.3(2334)$ \\
\hline Florfenicol & $1.6(9) *$ & $8.4(116)^{+}$ & $18.7(262)^{\#}$ & $11.5(387)$ & $4.5(41)^{*}$ & $45.8(712)^{\dagger}$ & $14.3(250)^{\#}$ & $23.8(1003)$ \\
\hline Gentamicin & $0.2(1)$ & $0.7(10)^{+}$ & $2.4(33)^{\#}$ & $1.3(44)$ & $7.5(68)^{*}$ & $18.9(294)^{\dagger}$ & $10.3(181)^{\#}$ & $12.9(543)$ \\
\hline Kanamycin & $6.3(36) *$ & $9.5(132)$ & $10.1(142)^{\#}$ & $9.2(310)$ & $13.4(122)^{*}$ & $44.3(690)^{\dagger}$ & $21.3(374)^{\#}$ & 28.1 (1186) \\
\hline Linezolid & $0.3(2)$ & $0(0)^{+}$ & $5.2(73)^{\#}$ & $2.2(75)$ & $0.2(2)^{*}$ & $1.4(22)$ & $1.1(20)^{\#}$ & $1.0(44)$ \\
\hline Quinupristin/dalfopristin & $8.7(50)$ & $11.9(165)^{\dagger}$ & $28.0(393)^{\#}$ & $18.1(608)$ & ND & ND & ND & ND \\
\hline Salinomycin & $0.2(1)$ & $0.4(5)^{\dagger}$ & $11.0(154)^{\#}$ & $4.8(160)$ & $0(0)$ & $0(0)^{\dagger}$ & $2.8(49)^{\#}$ & $1.2(49)$ \\
\hline Streptomycin & $6.8(39) *$ & $12.4(172)^{\dagger}$ & $30.8(432)^{\#}$ & $19.1(643)$ & $18.8(171)^{*}$ & $50.9(792)^{\dagger}$ & $31.8(558)^{\#}$ & 36.1 (1521) \\
\hline Tetracycline & $25.9(148)^{*}$ & $20.1(279)^{\dagger}$ & $61.7(866)^{\#}$ & $38.5(1293)$ & $41.8(380) *$ & $78.3(1219)$ & $76.1(1333)^{\#}$ & $69.5(2932)$ \\
\hline Tigecycline & $6.8(39)$ & $5.8(81)$ & $5.5(77)$ & $5.9(197)$ & $7.5(68)^{*}$ & $11.5(179)$ & $10.6(185)^{\#}$ & $10.2(432)$ \\
\hline Tylosin & $4.9(28) *$ & $13.8(191)^{\dagger}$ & $53.0(743)^{\#}$ & $28.6(962)$ & $20.1(183) *$ & 66.6 (1036) & $63.6(1115)^{\#}$ & $55.3(2334)$ \\
\hline Vancomycin & $0(0)$ & $0(0)$ & $0(0)$ & $0(0)$ & $0(0)$ & $0(0)$ & $0(0)$ & $0(0)$ \\
\hline MDR & $8.6(49) *$ & $15.6(216)^{\dagger}$ & $60.9(854)^{\#}$ & $33.3(1119)$ & $19.7(179)^{*}$ & $63.6(990)^{+}$ & $59.7(1046)^{\#}$ & $52.5(2215)$ \\
\hline
\end{tabular}

${ }^{*} p<0.05$ compared with the resistance rates in pigs, ${ }^{\#} p<0.05$ compared with the resistance rates in chickens, and ${ }^{+} p<0.05$ compared with the resistance rates in pigs.

E. faecium isolates recovered from chickens demonstrated a very high resistance rate to most of the tested antimicrobials compared with those isolated from cattle and pigs (Table 1). More than half of the E. faecium isolates from chickens were resistant to ciprofloxacin, erythromycin, tetracycline, and tylosin. E. faecium isolates from cattle presented relatively low resistance rates $(<10.0 \%)$ to the tested antimicrobials except to tetracycline $(25.9 \%)$, ciprofloxacin $(23.1 \%)$, and erythromycin (19.9\%). Similarly, pig isolates exhibited low resistance rates $(<15.0 \%)$ to most of the tested antimicrobials, except to erythromycin $(31.5 \%)$ and tetracycline $(20.1 \%)$. We observed linezolid $(0.0-5.2 \%)$, daptomycin $(5.6-11.1 \%)$, and tigecycline $(5.5-6.8 \%)$ resistance in a relatively small percentage of E. faecium isolates. Indeed, the linezolid resistance rate was high in chicken isolates $(5.2 \%)$ compared with those of cattle $(0.3 \%)$ and pigs $(0.0 \%)$. Additionally, we identified high-level gentamicin $(1.3 \%$, MIC $\geq 500 \mu \mathrm{g} / \mathrm{mL})$, kanamycin $(15.0 \%$, MIC $\geq 500 \mu \mathrm{g} / \mathrm{mL})$, and streptomycin $(17.1 \%, \mathrm{MIC} \geq 2000 \mu \mathrm{g} / \mathrm{mL})$ resistance in E. faecium isolated mainly 
from chickens and pigs (Supplementary Table S2). The $\mathrm{MIC}_{50}$ and $\mathrm{MIC}_{90}$ of the tested antimicrobials against E. faecium isolated from cattle, chickens, and pigs are summarized in Supplementary Table S3A-C.

E. faecalis isolated from chickens and pigs exhibited high resistance rate to most of the tested antimicrobials, especially to tetracycline (76.1-78.3\%), erythromycin (63.0-67.1\%), and tylosin (63.6-66.6\%) (Table 1). In contrast, cattle isolates presented a moderate or low resistance rate to these antimicrobials. We identified linezolid $(1 \%)$, daptomycin $(0.8 \%)$, and tigecycline $(10.2 \%)$ resistance in a very small proportion of E. faecalis isolates. High-level gentamicin (14\%), kanamycin $(28.5 \%)$, and streptomycin $(34.2 \%)$ resistances were noted in E. faecalis isolated predominantly from chickens and pigs (Supplementary Table S4). The $\mathrm{MIC}_{50}$ and $\mathrm{MIC}_{90}$ of the tested antimicrobials against E. faecalis isolated from cattle, chickens, and pigs are summarized in Supplementary Table S5A-C.

\subsection{Antimicrobial Resistance Trends}

We observed variations in the antimicrobial resistance trend between the two strains as well as in the same strain from different sources. E. faecium isolates exhibited an increasing but fluctuating trend of resistance to ciprofloxacin and tigecycline (isolates from cattle), erythromycin (from pigs), and daptomycin and linezolid (from chickens) (Figure 1 and Supplementary Tables S6-S8). In contrast, we noted a decreasing but fluctuating resistance trend to some of the tested antimicrobials in E. faecium isolated from cattle (tetracycline, streptomycin, and tylosin) and chickens (streptomycin, ciprofloxacin, tetracycline, erythromycin, tylosin, and quinupristin/dalfopristin). Although we did not find a significant change in the daptomycin resistance trend in E. faecium isolated from cattle and pigs, resistance rates peaked in 2016.
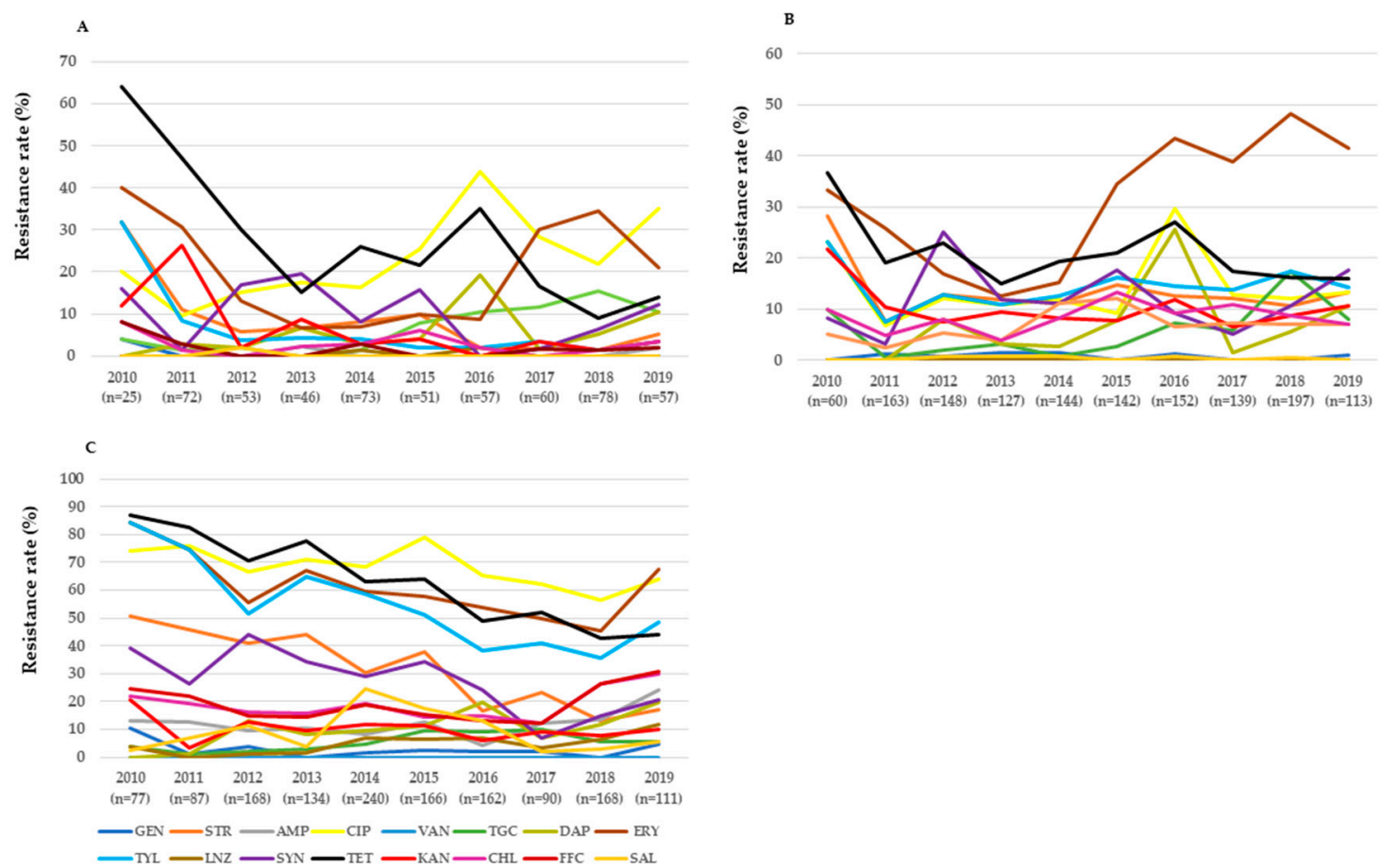

Figure 1. Antimicrobial resistance trend of E. faecium isolates recovered from cattle (A), pigs (B), and chickens (C) in Korea from 2010 to 2019. Abbreviations: AMP, ampicillin; CIP, ciprofloxacin; CHL, chloramphenicol; DAP, daptomycin; ERY, erythromycin; FFC, florfenicol; GEN, gentamicin; KAN, kanamycin; LIN, linezolid; SAL, salinomycin; STR, streptomycin; SYN, quinupristin/dalfopristin; TET, tetracycline; TGC tigecycline, TYL, tylosin; and VAN, vancomycin.

E. faecalis isolates presented an increasing but fluctuating resistance trend to tigecycline (isolates from cattle), erythromycin, tigecycline, linezolid, and florfenicol (from pigs), and florfenicol (from chickens) (Figure 2 and Supplementary Tables S9-S11). Neverthe- 
less, a decreasing but fluctuating resistance trend was noted in E. faecalis isolated from cattle (gentamicin, streptomycin, erythromycin, tylosin and tetracycline) and chickens (ciprofloxacin, erythromycin, tylosin and tetracycline). In addition, we noted a stable daptomycin resistance rate in E. faecalis isolated from cattle, chickens and pigs.
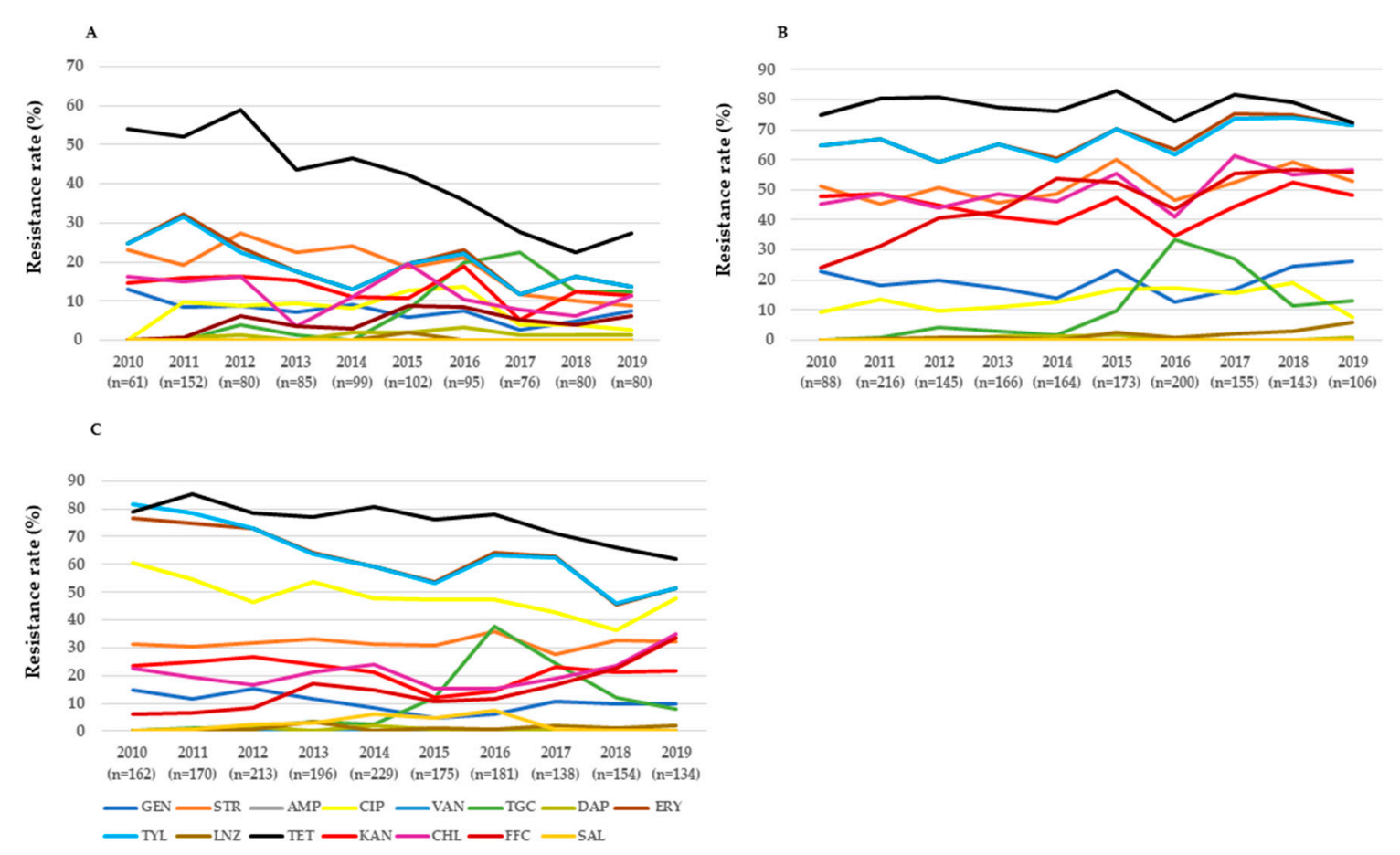

Figure 2. Antimicrobial resistance trend of E. faecalis isolates recovered from cattle (A), pigs (B), and chickens (C) in Korea from 2010 to 2019. Abbreviations: AMP, ampicillin; CIP, ciprofloxacin; CHL, chloramphenicol; DAP, daptomycin; ERY, erythromycin; FFC, florfenicol; GEN, gentamicin; KAN, kanamycin; LIN, linezolid; SAL, salinomycin; STR, streptomycin; TET, tetracycline; TGC, tigecycline, TYL, tylosin; and VAN, vancomycin.

\subsection{Multidrug Resistance (MDR) and Antimicrobial Resistance Patterns}

The majority of E. faecium (72.0\%) and E. faecalis (80.7\%) isolates were resistant to one or more of the tested antimicrobials (Tables 2 and 3). Notably, 33.3\% of the E. faecium and $52.5 \%$ of the E. faecalis isolates were resistant to multiple antimicrobials (Table 1). MDR was high in E. faecium isolated from chickens $(60.9 \%)$, and in E. faecalis recovered from pigs $(63.6 \%)$ and chickens $(59.7 \%)$. Besides, resistance to five or more antimicrobials was noted in E. faecium isolated from chickens (44.7\%), and in E. faecalis from pigs (56.6\%) and chickens $(34.7 \%)$ (Tables 2 and 3). A decreasing $(p<0.05)$ but fluctuating MDR trend was found in E. faecium and E. faecalis isolated from cattle and chickens, whereas the converse was noted in E. faecalis from pigs (Supplementary Tables S6, S8-S10 and S11).

A total of 434 and 262 MDR combination patterns were observed in E. faecium and E. faecalis isolates, respectively. Ciprofloxacin resistance with $(5.2 \%)$ or without (5.7\%) tetracycline was frequently noted in E. faecium isolated from chickens, whereas resistance to erythromycin was predominant in cattle $(11 \%)$ and pig $(12.9 \%)$ isolates (Table 2). Tetracycline resistance was frequently noted in E. faecalis isolated from cattle (14.5\%) (Table 3). Tetracycline resistance with $(11.0 \%)$ or without $(11.5 \%)$ ciprofloxacin and macrolides were most frequent in E. faecalis isolated from chickens. The most frequent MDR pattern in E. faecalis isolated from pigs was resistance to seven antimicrobials $(9.4 \%)$, including macrolides (Table 3). 
Table 2. Frequent resistance patterns in E. faecium isolates $(n=3360)$ recovered from cattle, pigs, and chickens between 2010 and 2019 in Korea.

\begin{tabular}{|c|c|c|c|}
\hline Animal Species & No. of Antimicrobials & No. of Isolates (\%) & Most Frequent Resistance Pattern \\
\hline \multirow[t]{11}{*}{ Cattle $(n=572)$} & 0 & $216(37.8)$ & \\
\hline & 1 & $199(34.8)$ & ERY $(n=63)$ \\
\hline & 2 & $107(18.7)$ & CIP TET $(n=43)$ \\
\hline & 3 & $22(3.8)$ & CHL STR TET $(n=3)$ \\
\hline & 4 & $7(1.2)$ & ERY STR TET TYL $(n=4)$ \\
\hline & 5 & $3(0.5)$ & $\begin{array}{l}\text { CIP ERY KAN TET TYL }(n=1) \text {, ERY KAN STR } \\
\text { TET TYL }(n=1) \text {, ERY SYN STR TET TYL }(n=1)\end{array}$ \\
\hline & 6 & $9(1.6)$ & CIP ERY KAN STR TET TYL $(n=3)$ \\
\hline & 7 & $4(0.7)$ & $\begin{array}{c}\text { AMP CHL CIP ERY STR TET TYL }(n=1) \text {, CHL } \\
\text { CIP FFC ERY STR TET TYL }(n=1) \text {, CHL FFC ERY } \\
\text { KAN STR TET TYL }(n=1), \text { CIP ERY KAN SYN } \\
\text { STR TET TYL }(n=1)\end{array}$ \\
\hline & 8 & $4(0.7)$ & $\begin{array}{c}\text { CHL CIP FFC ERY KAN STR TET TYL }(n=1), \\
\text { CHL CIP FFC ERY LZD STR TET TYL }(n=1), \text { CHL } \\
\text { CIP FFC ERY SYN STR TET TYL }(n=1), \text { CHL FFC } \\
\text { ERY KAN SYN STR TET TYL }(n=1)\end{array}$ \\
\hline & 9 & $0(0)$ & \\
\hline & 10 & $1(0.2)$ & $\begin{array}{l}\text { AMP CHL CIP FFC ERY KAN SYN STR TET TYL } \\
\qquad(n=1)\end{array}$ \\
\hline \multirow[t]{11}{*}{ Pigs $(n=1385)$} & 0 & $605(43.7)$ & \\
\hline & 1 & $406(29.3)$ & $\operatorname{ERY}(n=179)$ \\
\hline & 2 & $137(9.9)$ & CIP TET $(n=25)$ \\
\hline & 3 & $49(3.5)$ & ERY TET TYL $(n=8)$ \\
\hline & 4 & $26(1.9)$ & $\begin{array}{l}\text { CHL FFC ERY TYL }(n=3) \text {, CIP ERY TET TYL } \\
(n=3), \text { ERY STR TET TYL }(n=3)\end{array}$ \\
\hline & 5 & $44(3.2)$ & ERY KAN STR TET TYL $(n=9)$ \\
\hline & 6 & $56(4.0)$ & CHL FFC ERY STR TET TYL $(n=17)$ \\
\hline & 7 & $28(2.0)$ & CHL FFC ERY KAN STR TET TYL $(n=8)$ \\
\hline & 8 & $25(1.8)$ & CHL FFC ERY KAN SYN STR TET TYL $(n=13)$ \\
\hline & 9 & $6(0.4)$ & CHL CIP FFC ERY KAN SYN STR TET TYL $(n=4)$ \\
\hline & 10 & $3(0.2)$ & $\begin{array}{c}\text { AMP CHL CIP FFC DAP ERY SYN STR TET TYL } \\
(n=1), \text { CHL CIP FFC ERY GEN KAN SYN STR } \\
\text { TET TYL }(n=1), \text { CHL CIP FFC ERY KAN SYN } \\
\text { STR TET TGC TYL }(n=1)\end{array}$ \\
\hline \multirow[t]{12}{*}{ Chicken $(n=1403)$} & 0 & $134(9.6)$ & \\
\hline & 1 & $205(14.6)$ & $\mathrm{CIP}(n=80)$ \\
\hline & 2 & $158(11.3)$ & CIP TET $(n=73)$ \\
\hline & 3 & $132(9.4)$ & CIP STR TET $(n=24)$ \\
\hline & 4 & $146(10.4)$ & CIP ERY TET TYL $(n=53)$ \\
\hline & 5 & $195(13.9)$ & CIP ERY STR TET TYL $(n=48)$ \\
\hline & 6 & $191(13.6)$ & CIP ERY SYN STR TET TYL $(n=38)$ \\
\hline & 7 & $101(7.2)$ & AMP CIP ERY SYN STR TET TYL $(n=7)$ \\
\hline & 8 & $73(5.2)$ & CHL CIP FFC ERY SYN STR TET TYL $(n=12)$ \\
\hline & 9 & $51(3.6)$ & AMP CHL CIP FFC ERY SYN STR TET TYL $(n=8)$ \\
\hline & 10 & $16(1.1)$ & $\begin{array}{l}\text { AMP CHL CIP FFC ERY LZD SAL STR TET TYL } \\
\qquad(n=3)\end{array}$ \\
\hline & 11 & $1(0.1)$ & $\begin{array}{l}\text { CHL CIP FFC ERY KAN LZD SYN SAL STR TET } \\
\text { TYL }(n=1)\end{array}$ \\
\hline
\end{tabular}

Abbreviations: AMP, Ampicillin; CIP, Ciprofloxacin; CHL, Chloramphenicol; DAP, Daptomycin; ERY, Erythromycin; FFC, Florfenicol; GEN, Gentamicin; KAN, Kanamycin; LNZ, Linezolid; SAL, Salinomycin; STR, Streptomycin; SYN, Quinupristin/Dalfopristin; TET, Tetracycline; TYL, Tylosin; TGC, Tigecycline; VA, Vancomycin. 
Table 3. Frequent resistance patterns in E. faecalis isolates $(n=4218)$ recovered from cattle, pigs, and chickens between 2010 and 2019 in Korea.

\begin{tabular}{|c|c|c|c|}
\hline Animal Species & No. of Antimicrobials & No. of Isolates (\%) & Most Frequent Resistance Pattern \\
\hline \multirow[t]{10}{*}{ Cattle $(n=910)$} & 0 & $454(49.9)$ & \\
\hline & 1 & $184(20.2)$ & TET $(n=132)$ \\
\hline & 2 & $66(7.3)$ & STR TET $(n=32)$ \\
\hline & 3 & $46(5.1)$ & ERY TET TYL $(n=19)$ \\
\hline & 4 & $28(3.1)$ & CHL ERY TET TYL $(n=10)$ \\
\hline & 5 & $32(3.5)$ & ERY KAN STR TET TYL $(n=17)$ \\
\hline & 6 & $33(3.6)$ & ERY GEN KAN STR TET TYL $(n=7)$ \\
\hline & 7 & $47(5.2)$ & CHL ERY GEN KAN STR TET TYL $(n=24)$ \\
\hline & 8 & $18(2.0)$ & CHL CIP ERY GEN KAN STR TET TYL $(n=6)$ \\
\hline & 9 & $2(0.2)$ & $\begin{array}{l}\text { CHL CIP FFC ERY KAN LZD STR TET TYL }(n=1), \\
\text { CHL FFC DAP ERY GEN KAN STR TET TYL }(n=1)\end{array}$ \\
\hline \multirow[t]{11}{*}{ Pigs $(n=1556)$} & 0 & $216(13.9)$ & \\
\hline & 1 & $186(12.0)$ & TET $(n=139)$ \\
\hline & 2 & $79(5.1)$ & STR TET $(n=24)$ \\
\hline & 3 & $88(5.7)$ & ERY TET TYL $(n=45)$ \\
\hline & 4 & $104(6.7)$ & CHL ERY TET TYL $(n=20)$ \\
\hline & 5 & $133(8.5)$ & CHL FFC ERY TET TYL $(n=53)$ \\
\hline & 6 & $269(17.3)$ & CHL FFC ERY STR TET TYL $(n=67)$ \\
\hline & 7 & $248(15.9)$ & CHL FFC ERY KAN STR TET TYL $(n=147)$ \\
\hline & 8 & $156(10.0)$ & CHL FFC ERY GEN KAN STR TET TYL $(n=73)$ \\
\hline & 9 & $66(4.2)$ & CHL CIP FFC ERY GEN KAN STR TET TYL $(n=37)$ \\
\hline & 10 & $11(0.7)$ & CHL CIP FFC ERY GEN KAN STR TET TGC TYL $(n=6)$ \\
\hline \multirow[t]{11}{*}{ Chickens $(n=1752)$} & 0 & $144(8.2)$ & \\
\hline & 1 & $264(15.1)$ & TET $(n=202)$ \\
\hline & 2 & $143(8.2)$ & CIP TET $(n=39)$ \\
\hline & 3 & $222(12.7)$ & ERY TET TYL $(n=83)$ \\
\hline & 4 & $369(21.1)$ & CIP ERY TET TYL $(n=193)$ \\
\hline & 5 & $277(15.8)$ & CIP ERY STR TET TYL $(n=52)$ \\
\hline & 6 & $141(8.0)$ & CIP ERY GEN KAN TET TYL $(n=17)$ \\
\hline & 7 & $97(5.5)$ & CHL CIP ERY KAN STR TET TYL $(n=16)$ \\
\hline & 8 & $64(3.7)$ & CHL CIP ERY GEN KAN STR TET TYL $(n=23)$ \\
\hline & 9 & $24(1.4)$ & CHL CIP FFC ERY GEN KAN STR TET TYL $(n=14)$ \\
\hline & 10 & $7(0.4)$ & CHL CIP FFC ERY GEN KAN LZD STR TET TYL $(n=4)$ \\
\hline
\end{tabular}

Abbreviations: AMP, Ampicillin; CIP, Ciprofloxacin; CHL, Chloramphenicol; DAP, Daptomycin; ERY, Erythromycin; FFC, Florfenicol; GEN, Gentamicin; KAN, Kanamycin; LNZ, Linezolid; SAL, Salinomycin; STR, Streptomycin; TET, Tetracycline; TYL, Tylosin; TGC, Tigecycline; VA, Vancomycin.

\section{Discussion}

Knowledge of the distribution of antimicrobial-resistant bacteria in food animals and the food chain is vital for determining the potential risk to human health. Korea relies on testing isolates from food animals and foods of animal origin to determine the development and trends of antimicrobial resistance in the food chain. Consequently, this study provides a better understanding of the antimicrobial resistance profiles of the two most common enterococcal isolates recovered from healthy food animals slaughtered in Korea.

In this study, a considerable proportion of enterococcal isolates exhibited resistance to tetracycline and ciprofloxacin. Consistent with this study, high tetracycline resistance was reported in E. faecium and E. faecalis isolated from chickens and pigs in Korea [15,16,18,19,25], other Asian countries [13,26], Europe [27-29], and North America [30,31]. The ciprofloxacin resistance rate in E. faecium (67.9\%) and E. faecalis (48.6\%) isolated from chickens were higher than previous reports in Korea $[15,16,18,25]$ but contradict reports from other countries [27,28,30-33]. About $85-100$ tons of tetracyclines and $35-40$ tons of quinolones (mainly 
enrofloxacin for poultry) were sold annually for the livestock industry in Korea during the study period [34]. Thus, the widespread use of these antimicrobials in livestock production provides selective pressure and accelerates the emergence of resistant Enterococcus strains.

The chloramphenicol and florfenicol resistance in E. faecium isolated from chickens (18.7-18.8\%) and E. faecalis isolated from pigs (45.8-50.0\%) were higher than those described in previous studies in Korea $[15,18,25]$. We also observed an increasing florfenicol resistance trend in E. faecalis isolated from pigs and chickens. Several studies in Asia and Europe reported variable chloramphenicol resistance rates in E. faecium (8-53\%) and E. faecalis (16-53\%) isolated from cattle, pigs and chickens $[26,32,35,36]$. The average annual consumption of florfenicol in Korean livestock especially in chickens and pigs was increased by about $50 \%$ in the last five years compared to the amount during 2010-2014 [34]. Thus, the frequent use of florfenicol in food animals might select for chloramphenicol and florfenicol resistance. Our recent studies also identified the oxazolidinone and phenicol resistance genes (fexA, optrA, and poxtA) in Enterococcus strains recovered from food animals and their carcasses in Korea. The optrA and poxt $A$ genes were transferred to recipient strains from about $48 \%$ and $28 \%$ of optr $A$ and poxt $A$-carrying enterococcal strains, respectively. Further, the fexA gene was co-transferred with the optrA gene in all optrA-positive transconjugants $[17,37,38]$. Therefore, horizontal dissemination of phenicol resistance genes among enterococcal isolates might also contribute to the increase in chloramphenicol and florfenicol resistance.

Enterococcus strains identified in this study demonstrated a relatively low resistance rate to the tested aminoglycosides; except to kanamycin and streptomycin in E. faecalis isolated from pigs. Additionally, high-level gentamicin, kanamycin and streptomycin resistance were noted among the Enterococcus strains. Previous studies in Asian countries [13,18, $25,26]$ and Europe $[27,29,36]$ have reported highly variable gentamicin $(8-95 \%)$, kanamycin $(38-62 \%)$, and streptomycin (62-100\%) resistance rates in E. faecium and E. faecalis isolated from various food animals. Although enterococci are intrinsically resistant to clinically achievable concentrations of aminoglycosides, they are considered the antimicrobials of choice to treat human enterococcal infections when combined with cell wall inhibitors [4]. The widespread application of aminoglycosides in food animals (50, 71, and 296 tons in cattle, chickens and pigs, respectively, between 2010 and 2019) in Korea could be associated with the emergence of resistance [34]. Of note, the emergence of high-level aminoglycosideresistant strains cannot be ignored because it could threaten the existing efficacy of the broad-spectrum activity of aminoglycosides.

Resistance to penicillin may narrow the therapeutic options for enterococcal infections [39]. Consistent with previous reports in Korea [18,25] and other countries [10,13,31,35,40], 4.8\% of E. faecium isolates, especially those isolated from chickens, and $0.1 \%$ of $E$. faecalis were resistant to ampicillin. In contrast, previous studies have reported a relatively high ampicillin resistance in E. faecium from poultry in Germany (28\%) [27], and E. faecalis from pigs in Thailand (44\%) and Laos (12\%) [26]. Ampicillin and penicillin are the most active $\beta$-lactams against Enterococcus inhibiting the synthesis of peptidoglycan [41]. Although we do not have information about the resistance determinants, intrinsic tolerance to the action of $\beta$-lactamase in E. faecium is associated with the presence of a species-specific chromosomal gene, $p b p 5$, which encodes a class B penicillin-binding protein (PBP) with low affinity for ampicillin. Maintenance of peptidoglycan in the stationary phase mediated by an L,D-transpeptidase $\left(\mathrm{Ld}_{\mathrm{tfm}}\right)$ and overproduction of $\beta$-lactamase have also been implicated in ampicillin resistance in E. faecium [41]. Similarly, the overproduction of $\beta$-lactamase and point mutations of penicillin-binding protein (PBP4) have been shown to confer ampicillin resistance in E. faecalis [41,42].

Macrolide-lincosamide-streptogramin antibiotics constitute an alternative therapy for the treatment of insidious enterococcal infections [43]. We noted high erythromycin and tylosin resistance in E. faecium isolated from chickens and E. faecalis from pigs and chickens. Worryingly, despite fluctuations, we found an increasing erythromycin resistance trend in E. faecium and E. faecalis obtained from pigs. Consistent with this study, a 
considerable proportion of E. faecium and E. faecalis isolates obtained from food animals, especially chickens and pigs, in Korea demonstrated resistance to erythromycin (31-90\%) and tylosin (50-94\%) $[16,18,19]$. Our findings were also consistent with previous studies in Asia [26,40,44], Europe, and the United States [28,29,31-33]. Recently, the Korea Animal Health Products Association reported an increase in the annual sales of macrolides, especially tylosin, for livestock uses. The extensive use of macrolides (209 tons in pigs between 2010 and 2019), especially tylosin in Korean livestock husbandry can cause selective pressure and lead to resistance [34]. Cross-resistance between erythromycin and tylosin could also contribute to the increase in the proportion of macrolide-resistant isolates. Further, Noh et al. [15] and Yoon et al. [45] have identified the $\operatorname{erm}(A)$ and $\operatorname{erm}(B)$ genes in virulent and multi-resistant strains of E. faecalis from chickens in Korea, indicating the dissemination of macrolide resistance genes among enterococcal isolates.

The quinupristin/dalfopristin resistance rate in E. faecium isolated from chickens was consistent with Kim et al. [16] in Korea and Unal et al. [32] in Canada, but much lower than other studies reported in Canada (89.7\%) [30] and the United States (63\%) [31]. The quinupristin/dalfopristin resistance rate in E. faecium isolated from pigs and cattle was comparable to the findings of Ramos et al. [28] in Portugal. Quinupristin/dalfopristin is not approved for animal use in Korea. The use of virginiamycin, a streptogramin showing cross-resistance with quinupristin/dalfopristin, could be linked to the occurrence of quinupristin/dalfopristin resistance in E. faecium [46]. For E. faecalis, the situation appears different, because this bacterium is intrinsically resistant to quinupristin/dalfopristin through activity conferred by the expression of the lsa gene [39]. Quinupristin/dalfopristinresistant Enterococcus in food animals may play a role in the emergence of human infections through the food chain, indicating the need for continuous and careful monitoring.

We also investigated antibiotic susceptibility to four antibiotics, namely daptomycin, tigecycline, linezolid and vancomycin, representing four different classes and not registered for use in veterinary medicine in Korea [34]. Consistent with previous reports in Korea [16,17,37,38] and other countries [47-50], the occurrence of linezolid resistance is still rare among enterococcal isolates from food animals. The rarity of linezolid resistance among enterococci might be due to the fact that resistance develops as a spontaneous mutation in the multiple copies $23 S r R N A$ gene [48]. A small proportion $(\leq 10 \%)$ of $E$. faecium and E. faecalis isolates exhibited resistance to daptomycin and tigecycline, which are considered critical to humans. Daptomycin resistance in E. faecium and E. faecalis is typically coordinated by the three-component cell envelope stress response system, LiaFSR [51]. The daptomycin resistance rate found in this study agreed with previous reports in enterococcal isolates from ducks and food animal carcasses in Korea [17,38] and cattle in Australia [10]. However, the tigecycline resistance rate contradicts the above reports. Vancomycin resistance in E. faecium and E. faecalis isolated from food animals has been reported in many countries [52]. In this study; however, all of the enterococcal isolates were susceptible to vancomycin. Overall, enterococcal isolates resistant to newer and critically important antimicrobials might transfer to humans through the food chain and makes the treatment of MDR infections a daunting clinical challenge.

The majority of E. faecium and E. faecalis isolates were resistant to at least one antimicrobial agent, and numerous resistance patterns were found in both species. The MDR rates in E. faecium and E. faecalis isolated from pigs and chickens were higher than those found by Kwon et al. [18] in Korea. In contrast, the MDR rate in Enterococcus isolates from chickens, pigs, and cattle from this study contradicts various reports in Europe [29,32] and other Asian countries [13,26]. Consistent with Novais et al. [6], Kim et al. [16] and Kwon et al. [18], MDR patterns usually include tetracycline, erythromycin and/or ciprofloxacin. The occurrence of MDR in Enterococcus species might be related to the propensity of the bacteria to be involved in various forms of conjugation. This can lead to the widespread dissemination of resistance determinants through plasmids [53]. Additionally, the hardiness of enterococci species may likely contribute to resistance development by enhancing the survivability of MDR strains in the environment. This has the potential of enhancing 
transmission from animals to humans [53]. Multidrug-resistant enterococcal isolates pose a serious threat to public health as the same class of antibiotics is being used in the treatment of most bacterial diseases in humans.

In conclusion, we found enterococcal isolates that exhibited resistance to several antimicrobials, including those considered critical for humans. The occurrence of a high percentage of multidrug-resistant E. faecium and E. faecalis in food animals is alarming, especially given the fact that very few antimicrobial agents can be used to control enterococcal infection. Such resistance is likely to be passed from food animals to humans through the food chain. Therefore, the prudent use of antimicrobials in food animals will be crucial in limiting the public health hazards of Enterococcus in Korea.

Supplementary Materials: The following are available online at https:/ / www.mdpi.com/article / 10.3390/microorganisms9050925/s1. Table S1. E. faecium and E. faecalis isolates recovered from cattle, pigs, and chickens between 2010 and 2019 in Korea. Table S2. MIC distribution of the tested antimicrobials against E. faecium isolated from cattle, chickens, and pigs between 2010 and 2019 in Korea. Table S3. The $\mathrm{MIC}_{50}$ and $\mathrm{MIC}_{90}$ of the tested antimicrobials against E. faecium isolated from cattle (A), pigs (B), chickens (C) between 2010 and 2019 in Korea. Table S4. MIC distribution of the tested antimicrobials against E. faecalis isolated from cattle, chickens, and pigs between 2010 and 2019 in Korea. Table S5 A. The $\mathrm{MIC}_{50}$ and $\mathrm{MIC}_{90}$ of the tested antimicrobials against $E$. faecalis isolated from cattle (A), pigs (B), chickens (C) between 2010 and 2019 in Korea. Table S6. Antimicrobial resistance rate in E. faecium isolated from cattle between 2010 and 2019 in Korea. Table S7. Antimicrobial resistance rate in E. faecium isolated from pigs between 2010 and 2019 in Korea. Table S8. Antimicrobial resistance rate in E. faecium isolated from chickens between 2010 and 2019 in Korea. Table S9. Antimicrobial resistance rate in E. faecalis isolated from cattle between 2010 and 2019 in Korea. Table S10. Antimicrobial resistance rate in E. faecalis isolated from pigs between 2010 and 2019 in Korea. Table S11. Antimicrobial resistance rate in E. faecalis isolated from chickens between 2010 and 2019 in Korea.

Author Contributions: Conceptualization, S.-K.L., and M.H.K.; methodology, M.H.K., J.-H.C., H.Y.K., and D.C.M.; software, A.F.M., J.-H.C., and S.-J.K.; validation, A.F.M., S.-J.K.; and J.-H.C.; formal analysis M.H.K., and S.-J.K.; investigation, M.H.K., H.Y.K., H.-J.S., J.-H.C., A.F.M., and S.-J.K.; data curation, H.-J.S., D.C.M., S.-S.Y., and S.-J.K.; writing-original draft preparation, A.F.M., and M.H.K.; writing-review and editing, A.F.M., D.C.M., and S.-K.L.; supervision, S.-S.Y., and S.-K.L.; project administration, D.C.M., and H.Y.K.; funding acquisition; S.-K.L., and D.C.M. All authors have read and agreed to the published version of the manuscript.

Funding: This research was funded by the Animal and Plant Quarantine Agency, Ministry of Agriculture, Food, and Rural Affairs, South Korea, grant B-1543081-2017-24-01.

Institutional Review Board Statement: Not applicable.

Informed Consent Statement: Not applicable.

Data Availability Statement: The data that support the findings of this study are available from the corresponding author upon reasonable request.

Conflicts of Interest: The authors declare no conflict of interest. The funders had no role in the design of the study; in the collection, analyses, or interpretation of data; in the writing of the manuscript, or in the decision to publish the results.

\section{References}

1. Hammerum, A.M. Enterococci of animal origin and their significance for public health. Clin. Microbiol. Infect. 2012, 18, 619-625. [CrossRef]

2. Sparo, M.; Urbizu, L.; Solana, M.V.; Pourcel, G.; Delpech, G.; Confalonieri, A.; Ceci, M.; Sánchez Bruni, S.F. High-level resistance to gentamicin: Genetic transfer between Enterococcus faecalis isolated from food of animal origin and human microbiota. Lett. Appl. Microbiol. 2012, 54, 119-125. [CrossRef]

3. Hao, H.; Sander, P.; Iqbal, Z.; Wang, Y.; Cheng, G.; Yuan, Z. The risk of some veterinary antimicrobial agents on public health associated with antimicrobial resistance and their molecular basis. Front. Microbiol. 2016, 7, 1626. [CrossRef]

4. Torres, C.; Alonso, C.A.; Ruiz-Ripa, L.; León-Sampedro, R.; del Campo, R.; Coque, T.M. Antimicrobial resistance in Enterococcus spp. of animal origin. Microbiol. Spectrum. 2018, 6, ARBA 0032. 
5. Nilsson, O. Vancomycin-resistant enterococci in farm animals-occurrence and importance. Infect. Ecol. Epidemiol. $2012,2,16959$. [CrossRef]

6. Novais, C.; Freitas, A.R.; Silveira, E.; Antunes, P.; Silva, R.; Coque, T.M.; Peixe, L. Spread of multidrug-resistant Enterococcus to animals and humans: An underestimated role for the pig farm environment. J. Antimicrob. Chemother. 2013, 68, $2746-2754$. [CrossRef] [PubMed]

7. Tyson, G.H.; Nyirabahizi, E.; Crarey, E.; Kabera, C.; Lam, C.; Rice-Trujillo, C.; McDermott, P.F.; Tate, H. Prevalence and antimicrobial resistance of enterococci isolated from retail meats in the United States, 2002 to 2014. Appl. Environ. Microbiol. 2018, 84, e01902-17. [CrossRef]

8. Boehm, A.B.; Sassoubre, L.M. Enterococci as indicators of environmental fecal contamination. In Enterococci: From Commensals to Leading Causes of Drug-Resistant Infection; Gilmore, M.S., Clewell, D.B., Ike, Y., Shankar, N., Eds.; Eye and Ear Infirmary: Boston, MA, USA, 2014.

9. Foka, F.E.; Ateba, C.N.; Lourenco, A. Detection of virulence genes in multidrug-resistant enterococci isolated from feedlots dairy and beef cattle: Implications for human health and food safety. Biomed. Res. Int. 2019, 2019, 5921840.

10. Barlow, R.S.; McMillan, K.E.; Duffy, L.L.; Fegan, N.; Jordan, D.; Mellor, G.E. Antimicrobial resistance status of Enterococcus from Australian cattle populations at slaughter. PLoS ONE 2017, 12, e0177728. [CrossRef]

11. Bertelloni, F.; Salvadori, C.; Moni, A.; Cerri, D.; Mani, P.; Ebani, V.V. Antimicrobial resistance in Enterococcus spp. isolated from laying hens of backyard poultry flocks. Ann. Agric. Environ. Med. 2015, 22, 665-669. [CrossRef]

12. Delpech, G.; Pourcel, G.; Schell, C.; De Luca, M.; Basualdo, J.; Bernstein, J.; Grenovero, S.; Sparo, M. Antimicrobial resistance profiles of Enterococcus faecalis and Enterococcus faecium isolated from artisanal food of animal origin in Argentina. Foodborne Pathog. Dis. 2012, 9, 939-944. [CrossRef] [PubMed]

13. Liu, Y.; Liu, K.; Lai, J.; Wu, C.; Shen, J.; Wang, Y. Prevalence and antimicrobial resistance of Enterococcus species of food animal origin from Beijing and Shandong Province, China. J. Appl. Microbiol. 2013, 114, 555-563. [CrossRef]

14. Layton, B.A.; Walters, S.P.; Lam, L.H.; Boehm, A.B. Enterococcus species distribution among human and animal hosts using multiplex PCR. J. Appl. Microbiol. 2010, 109, 539-547. [PubMed]

15. Noh, E.B.; Kim, Y.B.; Seo, K.W.; Son, S.H.; Ha, J.S.; Lee, Y.J. Antimicrobial resistance monitoring of commensal Enterococcus faecalis in broiler breeders. Poult. Sci. 2020, 99, 2675-2683. [CrossRef]

16. Kim, Y.J.; Park, J.H.; Seo, K.H. Comparison of the loads and antibiotic-resistance profiles of Enterococcus species from conventional and organic chicken carcasses in South Korea. Poult. Sci. 2018, 97, 271-278. [CrossRef] [PubMed]

17. Tamang, M.D.; Moon, D.C.; Kim, S.R.; Kang, H.Y.; Lee, K.; Nam, H.M.; Jang, G.C.; Lee, H.S.; Jung, S.C.; Lim, S.K. Detection of novel oxazolidinone and phenicol resistance gene optrA in enterococcal isolates from food animals and animal carcasses. Vet. Microbiol. 2017, 201, 252-256. [CrossRef] [PubMed]

18. Kwon, K.H.; Hwang, S.Y.; Moon, B.Y.; Park, Y.K.; Shin, S.; Hwang, C.Y.; Park, Y.H. Occurrence of antimicrobial resistance and virulence genes, and distribution of enterococcal clonal complex 17 from animals and human beings in Korea. J. Vet. Diagn. Investig. 2012, 24, 924-931. [CrossRef]

19. Hwang, I.Y.; Ku, H.O.; Lim, S.K.; Park, C.K.; Jung, G.S.; Jung, S.C.; Nam, H.M. Species distribution and resistance patterns to growth-promoting antimicrobials of enterococci isolated from pigs and chickens in Korea. J. Vet. Diagn. Investig. 2009, $21,858-862$. [CrossRef]

20. Lim, S.K.; Kim, T.S.; Lee, H.S.; Nam, H.M.; Joo, Y.S.; Koh, H.B. Persistence of vanA-type Enterococcus faecium in Korean livestock after ban on avoparcin. Microb. Drug Resist. 2006, 12, 136-139. [CrossRef]

21. Dutka-Malen, S.; Evers, S.; Courvalin, P. Detection of glycopeptide resistance genotypes and identification to the species level of clinically relevant enterococci by PCR. J. Clin. Microbiol. 1995, 33, 24-27. [CrossRef]

22. Clinical and Laboratory Standards Institute. Performance Standards for Antimicrobial Susceptibility Testing: Twentieth Informational Supplement, CLSI Document M100; CLSI: Wayne, PA, USA, 2018.

23. National Antimicrobial Resistance Monitoring System. NARMS Integrated Report: The National Antimicrobial Resistance Monitoring System: Enteric Bacteria; U.S. Food and Drug Administration: Rockville, MD, USA, 2014.

24. Danish Integrated Antimicrobial Resistance Monitoring and Research Program (DANMAP). Use of Antimicrobial Agents and Occurrence of Antimicrobial Resistance in Bacteria from Food Animals, Food, and Humans in Denmark; DANMAP Annual Report; Denmark. 2016; pp. 1600-2032. Available online: https://backend.orbit.dtu.dk/ws/portalfiles/portal/140535625/ DANMAP_2016_LOW_241017.pdf (accessed on 12 January 2021).

25. Sung, C.H.; Chon, J.W.; Kwak, H.S.; Kim, H.; Seo, K.H. Prevalence and antimicrobial resistance of Enterococcus faecalis and Enterococcus faecium isolated from beef, pork, chicken, and sashimi. Korean J. Food Sci. Anim. Resour. 2013, 33, 133-138. [CrossRef]

26. Thu, W.P.; Sinwat, N.; Bitrus, A.A.; Angkittitrakul, S.; Prathan, R.; Chuanchuen, R. Prevalence, antimicrobial resistance, virulence gene, and class 1 integrons of Enterococcus faecium and Enterococcus faecalis from pigs, pork, and humans in Thai-Laos border provinces. J. Glob. Antimicrob. Resist. 2019, 18, 130-138. [CrossRef]

27. Maasjost, J.; Mühldorfer, K.; De Jäckel, S.C.; Hafez, H.M. Antimicrobial susceptibility patterns of Enterococcus faecalis and Enterococcus faecium isolated from poultry flocks in Germany. Avian Dis. 2015, 59, 143-148. [CrossRef] [PubMed]

28. Ramos, S.; Igrejas, G.; Capelo-Martinez, J.L.; Poeta, P. Antibiotic resistance and mechanisms implicated in fecal enterococci recovered from pigs, cattle, and sheep in a Portuguese slaughterhouse. Ann. Microbiol. 2012, 62, 1485-1494. [CrossRef] 
29. Šeputiene, V.; Bogdaite, A.; Ružauskas, M.; Sužiedeliene, E. Antibiotic resistance genes and virulence factors in Enterococcus faecium and Enterococcus faecalis from diseased farm animals: Pigs, cattle, and poultry. Pol. J. Vet. Sci. 2012, 15, 431-438.

30. Tremblay, C.L.; Letellier, A.; Quessy, S.; Boulianne, M.; Daignault, D.; Archambault, M. Multiple-antibiotic resistance of Enterococcus faecalis and Enterococcus faecium from cecal contents in broiler chicken and turkey flocks slaughtered in Canada and plasmid colocalization of tetO and ermB genes. J. Food Prot. 2011, 74, 1639-1648. [CrossRef]

31. Hayes, J.R.; English, L.L.; Carr, L.E.; Wagner, D.D.; Joseph, S.W. Multiple-antibiotic resistance of Enterococcus spp. isolated from commercial poultry production environments. Appl. Environ. Microbiol. 2004, 70, 6005-6011. [CrossRef]

32. Ünal, N.; Aşkar, Ş.; Yildirim, M. Antibiotic resistance profile of Enterococcus faecium and Enterococcus faecalis isolated from broiler cloacal samples. Turk. J. Vet. Anim. Sci. 2017, 41, 199-203. [CrossRef]

33. Jackson, C.R.; Lombard, J.E.; Dargatz, D.A.; Fedorka-Cray, P.J. Prevalence, species distribution, and antimicrobial resistance of enterococci isolated from US dairy cattle. Lett. Appl. Microbiol. 2011, 52, 41-48. [CrossRef]

34. Animal and Plant Quarantine Agency. Korean Veterinary Antimicrobial Resistance Monitoring System; APQA: Gimcheon, Korea, 2019.

35. Pesavento, G.; Calonico, C.; Ducci, B.; Magnanini, A.; Lo Nostro, A. Prevalence and antibiotic resistance of Enterococcus spp. isolated from retail cheese, ready-to-eat salads, ham, and raw meat. Food Microbiol. 2014, 41, 1-7. [CrossRef]

36. Kasimoglu-Dogru, A.; Gencay, Y.E.; Ayaz, N.D. Prevalence and antibiotic resistance profiles of Enterococcus species in chicken at slaughter level; absence of vanA and vanB genes in E. faecalis and E. faecium.. Res. Vet. Sci. 2010, 89, 153-158. [CrossRef] [PubMed]

37. Na, S.H.; Moon, D.C.; Kim, M.H.; Kang, H.Y.; Kim, S.J.; Choi, J.H.; Mechesso, A.F.; Yoon, S.S.; Lim, S.K. Detection of the phenicol-oxazolidinone resistance gene poxta in Enterococcus faecium and Enterococcus faecalis from food-producing animals during 2008-2018 in Korea. Microorganisms 2020, 8, 1839. [CrossRef]

38. Na, S.H.; Moon, D.C.; Choi, M.J.; Oh, S.J.; Jung, D.Y.; Kang, H.Y.; Hyun, B.H.; Lim, S.K. Detection of oxazolidinone and phenicol-resistant enterococcal isolates from duck feces and carcasses. Int. J. Food Microbiol. 2019, 293, 53-59. [CrossRef]

39. Hollenbeck, B.L.; Rice, L.B. Intrinsic and acquired resistance mechanisms in enterococcus. Virulence 2012, 3, 421-569. [CrossRef] [PubMed]

40. Usui, M.; Ozawa, S.; Onozato, H.; Kuge, R.; Obata, Y.; Uemae, T.; Ngoc, P.T.; Heriyanto, A.; Chalemchaikit, T.; Makita, K.; et al. Antimicrobial susceptibility of indicator bacteria isolated from chickens in Southeast Asian countries (Vietnam, Indonesia, and Thailand). J. Vet. Med. Sci. 2014, 76, 685-692. [CrossRef] [PubMed]

41. Miller, W.R.; Munita, J.M.; Arias, C.A. Mechanisms of antibiotic resistance in enterococci. Expert. Rev. Anti. Infect. Ther. 2015, 12, 1221-1236. [CrossRef] [PubMed]

42. Ono, S.; Muratani, T.; Matsumuoto, T. Mechanism of resistance to imipenem and ampicillin in Enterococcus faecalis. Antimicrob. Agents Chemother. 2005, 49, 2954-2958. [CrossRef]

43. Lazar, V.; Gheorghe, I.; Curutiu, C.; Savin, I.; Marinescu, F.; Cristea, V.C.; Dobre, D.; Popa, G.L.; Chifiriuc, M.; Popa, M.L. Antibiotic resistance profiles in cultivable microbiota isolated from some Romanian natural fishery lakes included in Nature 2000 network. BMC Vet. Res. 2021, 17, 1-11. [CrossRef]

44. Yoshimura, H.; Ishimaru, M.; Endoh, Y.S.; Kojima, A. Antimicrobial susceptibilities of enterococci isolated from feces of broiler and layer chickens. Lett. Appl. Microbiol. 2000, 31, 427-432. [CrossRef]

45. Yoon, S.; Son, S.H.; Kim, Y.B.; Seo, K.W.; Lee, Y.J. Molecular characteristics of optrA-carrying Enterococcus faecalis from chicken meat in South Korea. Poult. Sci. 2020, 99, 6990-6996. [CrossRef]

46. Simjee, S.; Jensen, L.B.; Donabedian, S.M.; Zervos, M.J. Enterococcus. In Antimicrobial Resistance in Bacteria of Animal Origin; Aarestrup, F.M., Ed.; ASM Press: Washington, DC, USA, 2006; pp. 315-328.

47. Mendes, R.E.; Hogan, P.A.; Jones, R.N.; Sader, H.S.; Flamm, R.K. Surveillance for linezolid resistance via the Zyvox(R) Annual Appraisal of Potency and Spectrum (ZAAPS) Programme (2014): Evolving resistance mechanisms with stable susceptibility rates. J. Antimicrob. Chemother. 2016, 71, 1860-1865. [CrossRef] [PubMed]

48. Wang, L.; He, Y.; Xia, Y.; Wang, H.; Liangm, S. Investigation of mechanism and molecular epidemiology of linezolid-resistant Enterococcus faecalis in China. Infect. Genet. Evol. 2014, 26, 14-19. [CrossRef]

49. Flamm, R.K.; Mendes, R.E.; Ross, J.E.; Sader, H.S.; Jones, R.N. An international activity and spectrum analysis of linezolid: ZAAPS Program results for 2011. Diagn. Microbiol. Infect. Dis. 2013, 76, 206-213. [CrossRef]

50. Patel, S.N.; Memari, N.; Shahinas, D.; Toye, B.; Jamieson, F.B.; Farrell, D.J. Linezolid resistance in Enterococcus faecium isolated in Ontario, Canada. Diagn. Microbiol. Infect. Dis. 2013, 77, 350-353. [CrossRef] [PubMed]

51. Prater, A.G.; Mehta, H.H.; Kosgel, A.J.; Miller, W.R.; Tran, T.T.; Arias, C.A.; Shamoo, Y. Environment shapes the accessible daptomycin resistance mechanisms in Enterococcus faecium. Antimicrob. Agents Chemother. 2019, 63, e00790-19. [CrossRef] [PubMed]

52. Arias, C.A.; Murray, B.E. The rise of the Enterococcus: Beyond vancomycin resistance. Nat. Rev. Microbiol. 2012, 10, 266-278. [CrossRef]

53. Gousia, P.; Economou, V.; Bozidis, P.; Papadopoulou, C. Vancomycin-resistance phenotypes, vancomycin-resistance genes, and resistance to antibiotics of enterococci isolated from food of animal origin. Foodborne Pathog. Dis. 2015, 12, 214-220. [CrossRef] 